\title{
Knowledge push based on design flow and user capacity model
}

\author{
Hao Jiang ${ }^{1, *}, P u$ Yin $^{1}$, Lin Guo ${ }^{1}$, Yibo Wang ${ }^{1}$ \\ ${ }^{1}$ Institute of Telecommunication Satellite, China Academy of Space Technology, Beijing 100094, China
}

\begin{abstract}
The knowledge demand of the designer is determined by the knowledge structure of the knowledge user and knowledge usage scenario. Therefore, this paper proposes a knowledge push method based on design activities workflow model and user capability model. By constructing the workflow model of design activities, this paper determines the designer's knowledge demand scenario. The relationship between design knowledge and design activity is determined by calculating the similarity between the label of the design activity and the key words of design knowledge. By constructing user capability model, we describe the designer's knowledge structure and design ability .This paper determine the designer's personalized knowledge needs according to the user capability model. Finally, this paper presents the prototype system and proves the feasibility of this method with the example of the part design.
\end{abstract}

\section{General Instructions}

With the trend of economic globalization and increasing competition, knowledge has been a vital factor for business operations in an enterprise ${ }^{[1]}$. With the development of information technology, a growing number of knowledge-intensive corporations have raised more concern about Knowledge Management (KM) ${ }^{[2]}$. Knowledge management becomes the key factor for knowledge sharing in an organization. One of the recent trends in knowledge management is the knowledge push. Knowledge push provides R4 Service: the Right information at the Right time in the Right way to the Right person ${ }^{[3]}$.

Many researchers pay close attention to knowledge management aiming at active personal knowledge push. $\mathrm{Xu}$ proposes a framework of an active knowledge service based on collaborative intent capture to assist product conceptual design ${ }^{[4]}$. Zhang proposes a framework of the knowledge push process including knowledge adapter and knowledge pusher based on contex $t^{[5-6]}$.Zhou presents the method of knowledge capture which based on ontology, and introduces the mechanism of knowledge pull and knowledge push ${ }^{[2]}$.

$\mathrm{Gu}$ Xingjiang proposes a knowledge push method based on ontology and rough set combining with the designer's knowledge demand is proposed to solve the problem of large amount of knowledge in design activities, knowledge dispersion and low efficiency of knowledge push ${ }^{[7]}$. Wang modeled the design intention and realized the accurate push of the design knowledge by establishing the mapping relation between the design knowledge and the design intention ${ }^{[8]}$. According to the difference of knowledge demand of designers, Feng yixiong puts forward a knowledge push method based on semantic feature analysis ${ }^{[9]}$. Xu rongzheng uses frequent pattern mining to analyze the corresponding situation of knowledge and design tasks, and pushes the design knowledge to the designer through similarity calculation [10]. Mo rong thinks that the change of processing characteristics in process manufacturing process is the carrier of process knowledge and proposes the method of knowledge representation and push of machining process driven by geometric variation ${ }^{[11]}$.

The current researchers have put forward a variety of knowledge matching methods to improve the accuracy of knowledge push. This paper argues that the key to improving the accuracy of knowledge push is to accurately grasp the knowledge demand of designers. The designer's knowledge needs are determined by two key factors: the current design task and the ability of the designer. The maximum set of knowledge requirements can be determined according to the current task. The set of knowledge that a designer needs to accomplish this task is a personalized subset of the maximum set. This paper proposed a knowledge push method based on design process and user capability model. First, we build the design activity workflow model and establish the mapping of design knowledge and design activities. Then, we build the user capability model to describe the designer's ability of design. Finally, the knowledge which is required by the designer is extracted from the knowledge set based on the user capability model.

\section{Design process and user capability model}


Accurate description of design task and user design ability is the basis of accurate design knowledge push 。 In order to realize the accurate push of design knowledge, we first need to model the design process and user ability.

\subsection{Design activities workflow model}

A design process is a collection of ordered activities which is organized in a certain sequence 。 Design process is the main process of designer's design activities. The knowledge push determines the designer's knowledge demand according to the designer's current design activities, and provides corresponding design knowledge for designers. Figure 1 is a typical design process. In order to complete the design task of the parts, we need to complete a series of design activities such as demand analysis, weight optimization, operational analysis, process analysis, interference inspection, and mechanical analysis. Analytica the knowledge requirements of each design activity and establish the relevance of design knowledge and design activities will be elaborated in the next section.

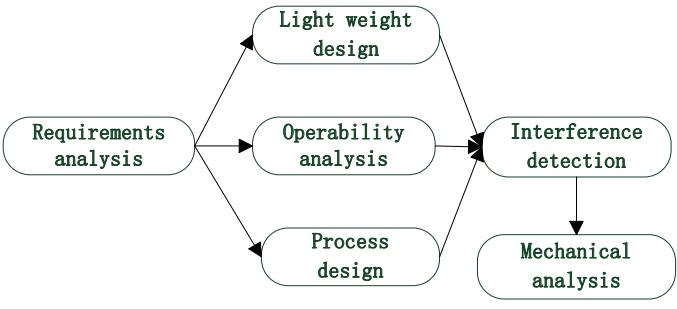

Fig. 1. Design activities workflow model.

\subsection{User capability model}

Even if the same design activity is completed, different designers have different design knowledge requirement due to their different knowledge structure and design ability. We need to provide personalized knowledge push service according to the designer's design ability. The designer's design ability is determined by his knowledge structure。This paper presents an extensible structured model of user capability to describe the designer's knowledge structure. Building user capability model is mainly for structured description the user's design knowledge structure, so its elements have a mapping relationship with the organization of knowledge. This paper constructs the basic framework of user capability model based on knowledge topic classification. The user capability model is shown in figure 2 .

This model divides the designer's mastery of the knowledge of a design domain into three situations: proficient, qualified and beginner. "Proficient ( P ) " means that the designer is an expert in the field of design. "qualified ( Q ) " indicates that the designer is a qualified designer in this field and has the ability to complete the design task in dependently. "beginner

(B) " indicates that the designer is a beginner in the field and has only a basic understanding of the field of design, which requires almost all design knowledge to complete the design task. This model allows you to extend it. As shown in figure 2, the parts design can be divided into mechanical analysis, weight optimization, process design and so on. The process design can be further refined into welding technology, machining technology, sheet metal process and casting process. Extensible user capability models allow designers to change and expand at any time. This feature allows users not to build a particularly accurate model at the beginning of their use. The designer can continuously improve the model which can improve the usability of the system.

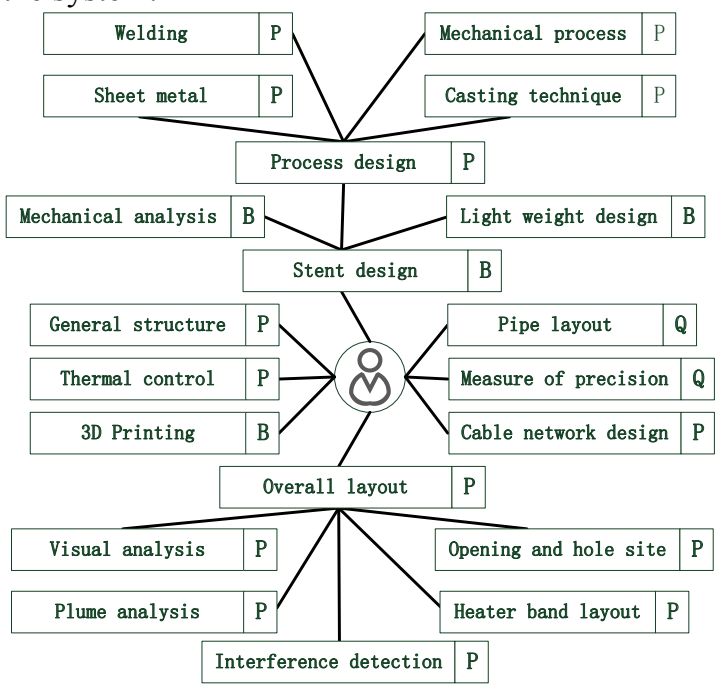

Fig. 2. User capability model.

\section{Personalized knowledge push}

After modeled the design process and user knowledge structure, it is possible to provide designers a set of personalized knowledge for their design tasks. In this paper, the knowledge push method based on the design process and user capability model firstly reorganizations the distributed knowledge in the field of knowledge. Then the knowledge requirement set is generated according to the knowledge requirement of the design activity, and the mapping of the knowledge requirement set and design activity is established. Finally, the personalized design knowledge requirement set is generated based on the user capability model, and it is pushed to the designer who is conducting the design activities. The process of knowledge push is shown in figure 3, mainly including knowledge reorganization, knowledge aggregation, knowledge linkage, knowledge matching and knowledge push.

\subsection{Knowledge reorganization}

At present, most enterprises construct various information systems, which lead to the design knowledge is stored in heterogeneous information systems. The design knowledge is stored in the enterprise's model base, document base, standard base, patent base, paper base, etc. When a designer needs to complete a design activity, it is necessary to find the design knowledge in multiple information systems, 
which greatly influences the design efficiency of the designer. In order to facilitate the retrieval and push of knowledge, this paper integrates distributed heterogeneous knowledge and reorganizes it according to the knowledge domain. A design domain contains all the knowledge of the field, including models and patents, standards, etc. The organization of design knowledge in the design field provides a good basis for the generation of design knowledge requirement set, because a design activity tends to correspond only to one design domain.

\subsection{Knowledge aggregation and knowledge linkage}

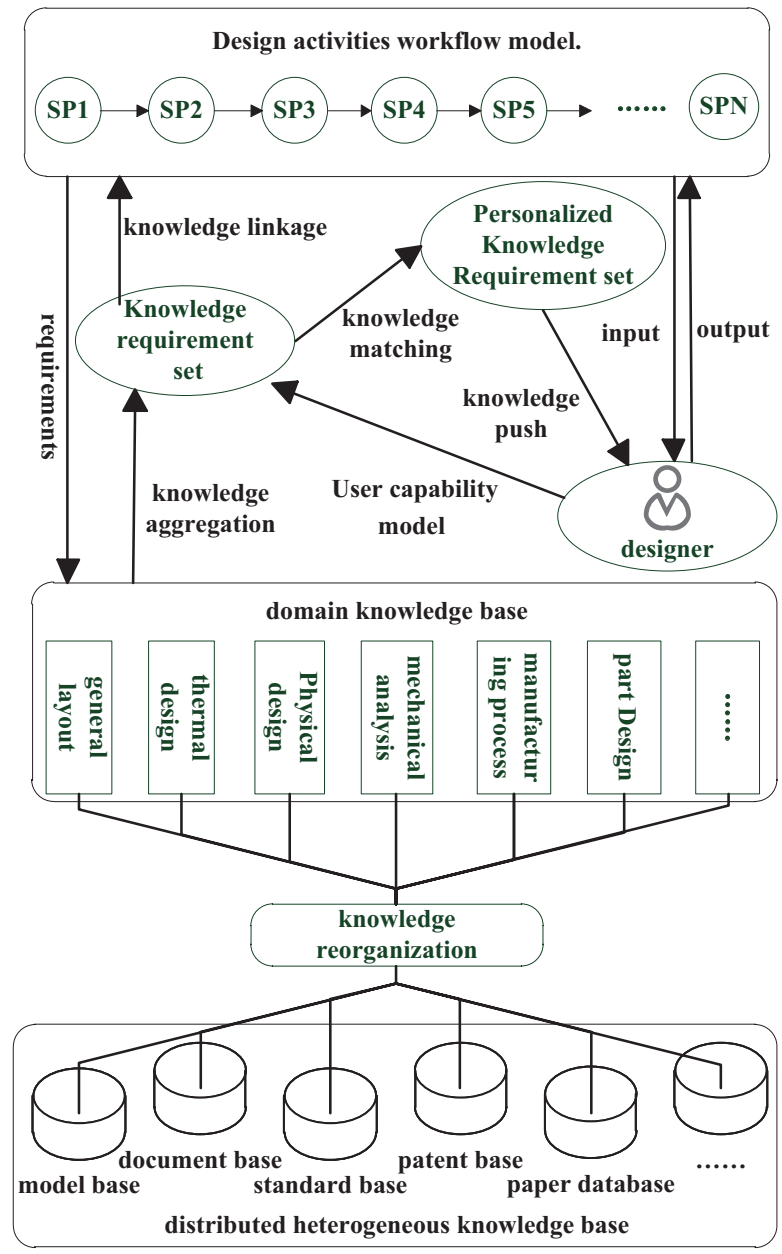

Fig. 3. Knowledge push control model.

Designers need different design knowledge to accomplish different design tasks. In order to realize the knowledge push for a certain design activity, it is necessary to retrieve specific series of design knowledge from the knowledge needs and characteristics of the design task. In this article, the designer has made a knowledge domain annotation for each design activity when building the design activities workflow model. We use multiple terms to describe the knowledge requirements for design activities. On the other hand, existing design knowledge also includes a set of keywords in its attributes. In this paper, the similarity of the design activity glossary and the design knowledge key word set is calculated by using the method of text similarity calculation. Then determine whether the design knowledge is required for this design activity based on the results of similarity calculation. The design activity glossary is $\mathrm{A}=\left\{a_{1}, a_{2}, a_{a}, \ldots, a_{n}\right\}$. The design knowledge key word set is $\mathrm{B}=\left\{b_{1}, b_{2}, b_{a}, \ldots, b_{m}\right\}$. The similarity calculation formula is as follows:

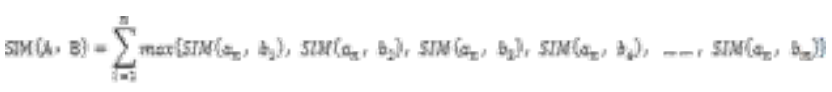

\section{3 knowledge matching and knowledge push}

The collection of knowledge requirements for specific design activities cannot be directly pushed to the designer. This collection is all the knowledge required for the design activity. Most designers don't need so much knowledge. We need to extract a set of personalized knowledge from this collection based on the user capability model of each designer. In the user capability model, designers are divided into three levels: "Proficient (P) " 、 "qualified ( Q ) "and "beginner (B) " in each design domain. This article determines whether the knowledge should be pushed to a specific designer based on the number of times the design knowledge is retrieved. This article pushes all design knowledge to the designer who is at the "beginner (B) " level. This article pushes the first $50 \%$ of the design knowledge to the designer who is at the "qualified (Q)

" level. This article pushes the first $20 \%$ of the design knowledge to the designer who is at the "Proficient ( P ) " level. Finally, the generated personalized knowledge requirement set is pushed to the designer.

\section{Application examples}

We developed the prototype system based on the knowledge push method which is based on the design process and user capability mode. Take a part design as an example to show the result of knowledge push. After combing the parts design process, the design process model is constructed, as shown in figure 4. To complete the design task of the parts, we need to complete a series of design activities in an orderly manner. The knowledge requirements set of process design contains four principles: 1 Size marking should be marked with the design reference and the process reference; 2 The shape of the parts is as simple as possible; 3 considering the accessibility of machining tools; 4 Avoid deep hole. Figure 5 is the push result of designers who is at the "qualified (Q) " level. Figure 6 is the push result of designers who is at the "beginner (B) " level. 


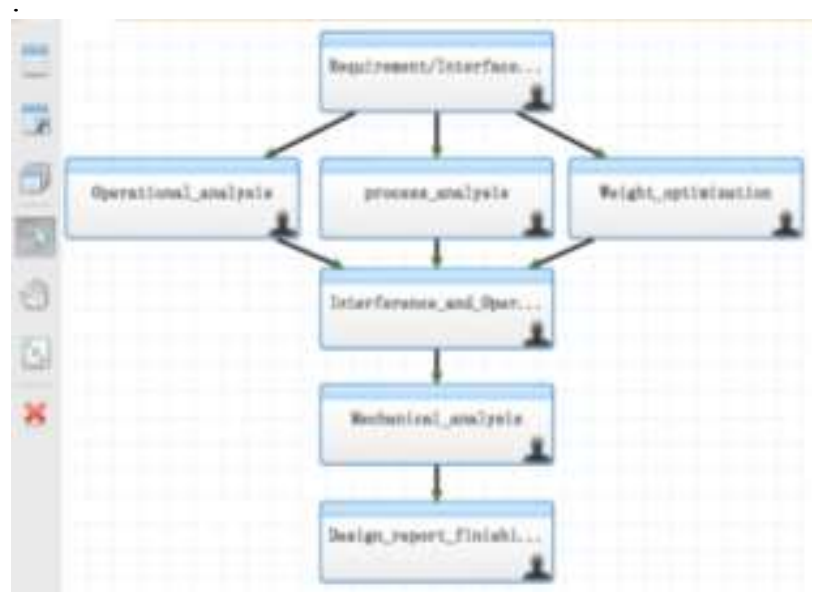

Fig. 4. Parts design process.

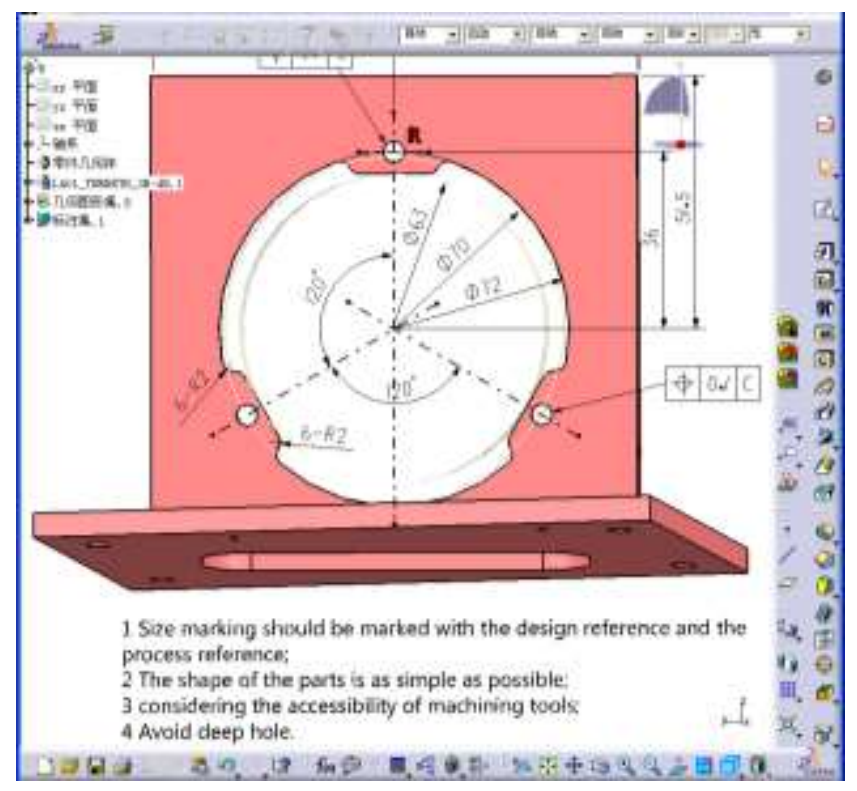

Fig. 5. Knowledge push for beginner.

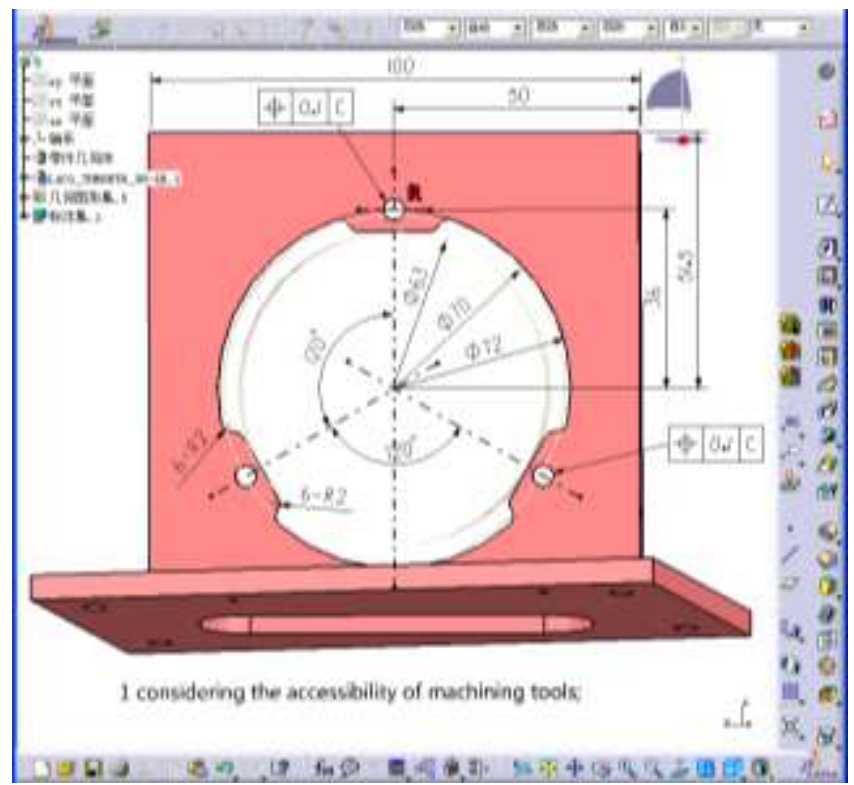

Fig. 6. Knowledge push for Proficient.

\section{5 conclusion and adaptation prospect}

This paper introduces the knowledge push method based on design process and user capability model. Constructing the design activities workflow model and user capability model are the important foundations for realizing the knowledge push. By constructing the design activities workflow model, the application scenario of design knowledge can be determined, which can help to determine the designer's knowledge demand for the design task. Extensible user capability model structured describe user knowledge structure and design capabilities. Its expandable features greatly improve the usability of the system. Finally, this paper presents the results of the knowledge push and proves the feasibility of this method with the example of the part design. How to build a more accurate correlation between design knowledge and design activities will be the next research focus. Automatic construction of user capability model will be another research focus.

\section{ACKNOWLEDGMENT}

National Key Technology Research and Development Program of the Ministry of Science and Technology of China (2015BAF18B01)

\section{References}

1. M.H.Shi, T.Wu, S.H.Jiang, Q.F.Wang, Y.D.Cheng, A Study of Knowledge Push System Based on Collaboration [A]. In: Proceeding of 2010 International Conference on Artificial Intelligence and Computational Intelligence [C],Nanjing,China,28-32,(2010)

2. M.J.Zhou, J.C.Tao, A Framework for OntologyBased Knowledge Management [A]. In: Proceeding of 2011 Business Management and Electronic Information [C], Guangzhou,China,428-431,( 2011)

3. Z.Y.Xin, J.Z.Zhao, C.H.Chi, J.G.Sun,. Information program generation for Webcasting [J].Data \& Knowledge Engineering, 49(1),1-21, (2004)

4. Y.H.Xun, G.F.Yin, Y.Nie, Z.W.He, X.L.Dai, Research on an active knowledge push service based on collaborative intent capture [J]. Journal of Network and Computer Applications, 36, 1418-1430, (2013).

5. F.P.Zhang, L.Li, Research on Knowledge Push Method for Business Process Based on Multidimensional Hierarchical Context Model [A]. In: Proceeding of 2016 IEEE International Conference on Industrial Engineering and Engineering management [C], Bali, Indonesia, 656660, (2016).

6. F.P.Zhang, L.Li, Research on Knowledge Push Method for Business Process Based on Multidimensional Hierarchical Context Model [J]. Journal of Computer-Aided Design \& Computer Graphics,29(4),751-758,(2017) 
7. X.Ji, X.J.GU, F.Dai, C.Y.Le, F.Y.Xu, Technology for product design knowledge push based on ontology and rough sets $[\mathrm{J}]$. computer Integrated Manufacturing Systems, 19(1), 7-20, (2013)

8. Z.S.wang, L.Tian, W.R.Duan, Knowledge push technology based on design intent modeling [J]. computer Integrated Manufacturing Systems , 21(3), 606-617, (2015)

9. Y.X.Feng, S.Y.Zhang, Y.C.Gao, J.Cheng, J.R.Tan, Intelligent push method of $\mathrm{CNC}$ design knowledge based on feature semantic analysis [J]. computer Integrated Manufacturing Systems , 22(1), 189-201, (2016)

10. R.Z.Xu, Q.Gao, H.Wang, T.Xu, Product design knowledge recommendation based on sequential pattern mining $[\mathrm{J}]$. computer Integrated Manufacturing Systems , 22(1), 189-201, (2016)

11. C.L.Li, R.Mo, Z.Y.Chang, H.C.Yang, D.L.Zhang, Y,Xiang, Knowledge representation and push of machining process driven by geometric variation [J]. computer Integrated Manufacturing Systems , 22(6), 1434-1446, (2016) 